\title{
为超疏水表面“披上”铠甲
}

王德辉, 于凡斐, 邓旭 ${ }^{*}$

电子科技大学基础与前沿研究院, 成都 610054

* 联系人, E-mail: dengxu@uestc.edu.cn

\section{Design of the armour for superhydrophobic surface}

\author{
Dehui Wang, Fanfei Yu \& Xu Deng ${ }^{*}$ \\ Institute of Fundamental and Frontier Sciences, University of Electronic Science and Technology of China, Chengdu 610054, China \\ * Corresponding author, E-mail: dengxu@uestc.edu.cn \\ doi: 10.1360/TB-2020-0922
}

超疏水表面因其自清洁、低固-液黏附、保持干燥等 多重功能特性，在生物技术、生物医学和传热传质等领域 展现出了巨大的应用前景 ${ }^{[1-5]}$. 水滴置放到超疏水表面时, 将呈现出较大的静态接触角 $\left(>150^{\circ}\right)$ 和较小的滚转角 $\left(<10^{\circ}\right)$. 一般情况下, 实现超疏水性需要结合低表面能和微/纳米 粗粘结构截留空气并托起液滴, 实现 Cassie-Baxter 态的同 时创造低的固-液接触. 然而, 在外部机械载荷下, 表面结 构会产生很高的局部压强, 使其极度脆弱, 易被磨损. 此 外, 磨损导致本体材料暴露, 表面由疏水变为亲水, 引起 超疏水性失效 ${ }^{[6]}$. 长期以来, 人们认为表面的机械稳定性 和超疏水性是两个相互排斥的性质, 正所谓“鱼和熊掌不 可兼得”. 针对上述问题, 我们在表面构造两种不同尺度的 结构来实现其机械性能的提升, 其中纳米结构提供排斥液 体的功能, 而微结构则用于保证表面的机械稳定性. 微结 构由类似于 “口袋” 的倒多棱雉为结构单元组成的阵列, 构 成一个相互连接的框架提供“铠甲”的作用, 从而避免“口 袋” 内超疏水但机械性能较差的纳米结构遭受磨损. 我们 将这一策略应用到硅、陶瓷、金属和透明玻璃等各类基底, 即使在砂纸和不锈钢刀片磨损后, 表面仍能维持优异的超 疏水性. 结果还表明, 拥有超强机械稳定性且高透光率的 自清洁玻璃有助于解决因灰尘污染引起太阳能电池效率 下降的问题. 我们的设计策略对于在恶劣环境中需保持高 效的自清洁、生物防污或强化传热传质等性能的材料开发 意义重大, 该研究成果发表于 Nature $e^{[7]}$.

通常, 减小固-液接触是提高表面超疏水性的常用手 段, 根据 Cassie-Baxter 方程, 固-液接触面积的减小, 有利 于提高表观接触角和降低滚动角. 但由于接触面积的降低, 必然导致微/纳结构承受更高的局部压强, 从而更易磨损, 这就意味着超疏水性和机械稳定性在提高一种性能时必
然导致另一种性能下降. 目前, 已有大量研究试图解决这 个问题, 例如，引入黏结层来加强超疏水层和基底之间的 结合力、通过随机引入离散的微结构来承受磨损、以及通 过牺牲上层自相似结构来换取超疏水性的维持. 然而, 上 述方法没解决根本问题, 机械性能只获得了一定程度的改 善. 不同于常规方法, 我们通过去耦合机制将超疏水性和 机械稳定性拆分至两种不同的结构尺度, 提出以微结构作 为 “铠甲”保护超疏水纳米材料免遭摩擦磨损的概念, 如图 1(a)所示. 作为设计的第一个特征, 微结构由一个结构连 续的框架构成, 可以更好地阻止所有尺寸大于微结构单元 的物体破坏填充在其内部的纳米材料, 如图 1(b), (c)所示. 另外, 基于对跳虫皮肤纹理和蜂房结构的理解, 连续结构 的机械稳定性在很大程度上要优于随机或离散结构. 与此 同时，铠甲的设计还必须考量微结构引人对表面润湿性的 影响. 我们通过 Cassie-Baxter 模型来分析固-液接触面积分 数 $f$ 、杨氏接触角 $\theta_{\mathrm{Y}}$ 和表观接触角 $\theta^{*}$ 之间的关系. 液滴在表 面呈稳定的 Cassie-Baxter 状态时, 以 $\theta_{\mathrm{Y}}$ 为系数, 可以绘制 出 $\theta^{*}$ 和 $f$ 之间的关系曲线(图 1(d)). 可以看出, 表面疏水(如 $\theta_{\mathrm{Y}}=120^{\circ}$ ) 和亲水 (如 $\theta_{\mathrm{Y}}=0^{\circ}$ ) 所对应的 $\theta^{*}$ 之间的差值 $\left(\Delta \theta^{*}\right)$ 随 $f$ 减小而迅速降低. 这表明, 在 $f$ 较小时, 材料表面本身的化 学性质对超疏水性能的影响越小. 也就是说, 只要将微结 构铠甲保持较低的 $f$, 即使表面经磨损从疏水变为亲水, 表 面的超疏水性能仍然不会发生较大改变.

通常情况下, 微观结构的力学稳定性受其几何形状影 响, 增加侧壁角度 $\alpha$ 是提高结构稳定性的有效方法. 为了 验证这一原理, 我们通过有限元建模分析了不同侧壁角度 微结构的稳定性, 证实了微观结构的稳定性随 $\alpha$ 的增大而 显著提高(图 1(f)). 相反, 若磨损使微结构发生断裂性损坏, 假设高度被磨损至 $1 / 2$ 时, 微结构的固-液接触分数变化量 
(a)

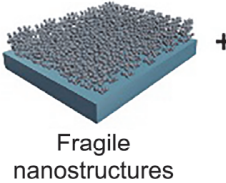

nanostructures

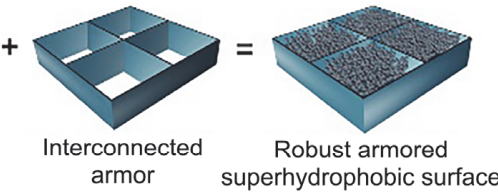

(c)

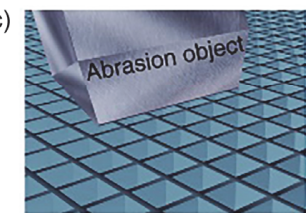

(e)

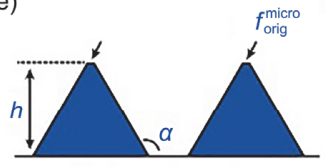

Before abrasion

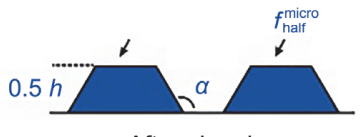

After abrasion (d)

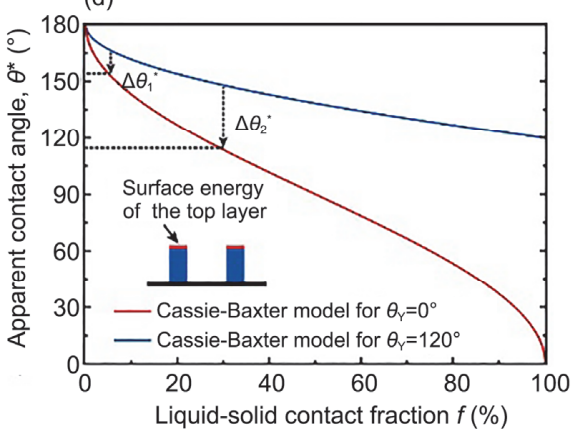

(f)

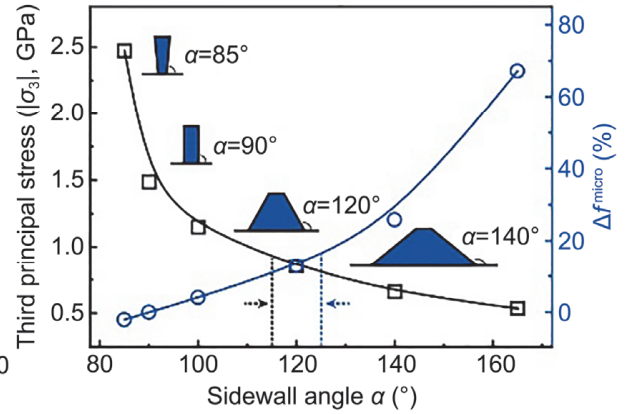

图 1 (网络版彩色)微结构铠甲的设计. (a) 为超疏水纳米材料披上保护性微结构“铠甲”来增强超疏水表面机械稳定性的策略; (b, c) 离 散和连续微结构表面的摩擦磨损示意图; (d) 在两种不同杨氏接触角 $\theta_{Y}$ 时, 表面液滴 Cassie-Baxter 状态下的表观接触角 $\theta^{*}$ 和固-液接触分 数 $f$ 之间的关系; (e) 连续框架结构高度 $h$ 因磨损降低至原始的 $1 / 2$ 时, 顶部接触面积的变化; (f) 微结构侧壁角度 $\alpha$ 与机械稳定性和固-液 接触分数变化量 $\Delta f$ 之间的函数关系 ${ }^{[7]}$

Figure 1 (Color online) Design of the microstructure armour. (a) The strategy for enhancing the mechanical stability of the superhydrophobic surface by housing water-repellent nanostructures within a protective microstructure "armour". (b, c) Schematics showing the mechanism by which abrasion damages discrete and interconnected microstructures. (d) Relationship between the apparent contact angle $\theta^{*}$ and the liquid-solid contact fraction $f$ for an ideal Cassie-Baxter state at two different values of the Young's contact angle $\theta_{\mathrm{Y}}$. (e) Cross-section showing the change of contact area on the top of the framework structures when the height $h$ is fractured to half of its original value by abrasion. (f) Influence of mechanical stability and change of the liquid-solid contact fraction $\Delta f^{\text {micro }}$ as function of the sidewall angle $(\alpha)^{[7]}$

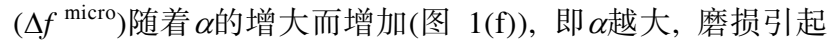
的超疏水性减弱越明显. 在 $\alpha \approx 120^{\circ}$ 时, 超疏水性和机械稳 定性可实现较好的平衡. 因此, “铠甲”策略的第二、第三个

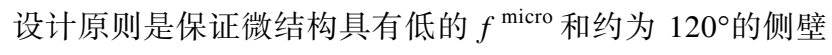
角度.

根据上述 3 个设计原则, 首先设计出倒四棱雉微腔阵 列构成的连续框架. 利用光刻、冷/热压等微细加工技术将 铠甲结构制备于硅片、陶瓷、金属、玻璃等普适性基材, 这 种方法也可应用于曲面基底, 并可通过卷对板印刷技术进 行大面积制造. 微结构“铠甲”与超疏水纳米材料简单复合, 即可构建出具有优良机械稳定性的锃甲化超疏水表面. 经 过不锈钢刀片的反复刮擦, 铠甲化表面表现出对垂直载荷 和剪切力良好的抵抗能力, 且填充在微结构内部的纳米材 料完好无损. 此时, 铠甲微结构顶部的疏水层已被磨损, 从疏水 $\left(\theta_{\mathrm{Y}} \approx 115^{\circ}\right)$ 变为亲水 $\left(\theta_{\mathrm{Y}} \approx 45^{\circ}\right)$. 通过激光共聚焦显微 镜三维成像观察磨损后的铠甲化表面, 其三相接触线得到 了填充超疏水纳米结构的有力支撑, 呈现出稳定的气-液固复合界面. 免遭磨损的纳米材料可以有效防止 Laplace
压力引起的气-液界面滑移, 使整个体系处于受约束的 Cassie-Baxter 平衡状态. 为了系统地评估磨损对于铠甲化 表面超疏水性能的影响, 我们设计了一系列具有不同微结 构尺寸和面积分数的超疏水表面, 并系统地测量了磨损前 后表面的静态接触角 $\theta^{*}$ 和滚动角 $\theta_{\text {roll-off }}$. 结果表明, 当 $f^{\text {micro }}$ 低于 $8 \%$ 时, 铠甲化表面在磨损后仍能保持超疏水性 $\left(\theta^{*}>150^{\circ}, \theta_{\text {roll-off }}<10^{\circ}\right)$. 同时, 该结果也验证了随着微结构 固-液接触分数的降低, 摩擦磨损对表面浸润性的影响随 之减弱这一基础理论，也说明表面的超疏水性与倒四棱雉 微结构的尺寸无关. 然而, 若发生了高度相同的破坏性磨 损，铠甲微结构的尺寸越小，固-液接触分数的变化 $\left(\Delta f^{\text {micro }}\right)$ 就越大, 其超疏水性对磨损越敏感. 因此, 合适的微结构 尺寸需要根据不同的应用场景作相应的调整. 我们还使用 液滴黏附力扫描显微镜(SDAM)测量了具有不同面积分数 表面 $\left(f^{\text {micro }} \approx 2 \%\right.$ 和 $\approx 7.8 \%$ )在磨损前后的固-液黏附力. 结果 表明, 磨损破坏了微结构顶部疏水层, 导致黏附力增加,

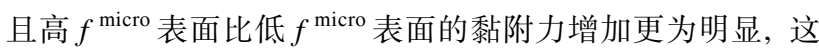
与 $\theta_{\text {roll-off }}$ 的变化趋势一致. 在分析磨损对表面射流冲击和 
水滴撞击实验的影响时, 得到了类似的结果, 因此, 只要 合理控制铠甲结构的面积分数, 就能很好地调控摩擦磨损 对表面浸润性的影响, 可有效地降低或避免因表面化学性 质被改变引起的超疏水性失效.

为了证明连续且具有较大侧壁角度的微结构框架可以 作为普适性的策略来实现表面机械稳定性的提升, 我们在 硅、金属及陶瓷基材上制备了倒三棱雉和倒六棱雉结构. 有 限元分析表明, 倒三棱雉和倒六棱雉结构具有与倒四棱雉 相似的力学原理和机械稳定性. 同时, 经摩擦磨损测试后, 也展现出了与倒四棱雉铠甲化表面相似的浸润性特点.

在实际应用中, 表面的耐磨性考验不可避免. 因此, 我们测试了铠甲化超疏水表面的长效机械耐久性, 并与普 通超疏水表面进行了对比. 以聚丙烯片作为磨损头对铠甲 化表面进行循环线性磨损. 经过 1000 次线性磨损后, 表面 仍然保持静态接触角大于 $150^{\circ}$ 和滚动角小于 $12^{\circ}$, 微结构 很好地抵抗了剪切力, 并有效保护了内部的纳米材料, 使 铠甲化超疏水表面的耐磨次数比传统超疏水表面高出 10
倍. 在胶带剥离、ASTM Taber 磨耗测试中, 铠甲化超疏水 表面同样表现出优异的机械稳定性. 此外, 我们还进行了 热稳定性 $\left(100^{\circ} \mathrm{C}\right.$ 暴露 $16 \mathrm{~d}$ )、化学腐蚀(浸泡在王水或 2.5 $\mathrm{mol} / \mathrm{L} \mathrm{NaOH}$ 溶液中 $4 \mathrm{~h}$ )、高速射流冲击(速度为 $32.6 \mathrm{~m} / \mathrm{s}$, 韦伯数 $\approx 36478$ ) 和高湿度环境中的冷凝测试等苛刻的耐久 性试验. 结果均表明, 面对上述极端恶劣条件, 我们的铠 甲化表面仍然能够保持优异的超疏水性能.

综上所述, 通过引入“铠甲”概念来提高超疏水表面机 械稳定性的策略切实可行, 这得益于对表面超疏水性和机 械稳定性拆分，使机械稳定性、超疏水性和高透光率得到 了有效平衡. 采用这一策略, 我们为太阳能电池构筑了一 种耐磨且高透光率的自清洁表面. 与传统的清洁过程相比, 这种表面能够借助雨或冷凝液滴被动去除灰尘污染，保持 电池较高的能量转换效率, 节省传统清洁过程中必需的淡 水资源和劳动力成本. 该论文创新的设计思路和通用的制 造策略展示了铠甲化超疏水表面非凡的应用潜力, 必将进 一步推动超疏水表面进人广泛的实际应用.

\section{推葆阅读文献}

1 Feng L, Li S H, Li Y S, et al. Super-hydrophobic surfaces: From natural to artificial. Adv Mater, 2002, 14: 1857-1860

2 Zheng Y M, Bai H, Huang Z B, et al. Directional water collection on wetted spider silk. Nature, 2010, 463: 640-643

3 Quéré D. Wetting and roughness. Annu Rev Mater Res, 2008, 38: 71-99

4 Sun Q Q, Wang D H, Li Y N, et al. Surface charge printing for programmed droplet transport. Nat Mater, 2019, 18: 936-941

$5 \mathrm{Xu} \mathrm{W} \mathrm{H,} \mathrm{Zheng} \mathrm{H} \mathrm{X,} \mathrm{Liu} \mathrm{Y,} \mathrm{et} \mathrm{al.} \mathrm{A} \mathrm{droplet-based} \mathrm{electricity} \mathrm{generator} \mathrm{with} \mathrm{high} \mathrm{instantaneous} \mathrm{power} \mathrm{density.} \mathrm{Nature,} \mathrm{2020,} \mathrm{578:}$ 392-396

6 Tian X L, Verho T, Ras R H A, et al. Moving superhydrophobic surfaces toward real-world applications. Science, 2011, 352: 142-143

7 Wang D H, Sun Q Q, Hokkanen M J, et al. Design of robust superhydrophobic surfaces. Nature, 2020, 582: 55-59 